\title{
ON THE NULL-CONTROLLABILITY OF DIFFUSION EQUATIONS
}

\author{
Gérald Tenenbaum ${ }^{1}$ and Marius TuCsnaK ${ }^{1}$
}

\begin{abstract}
This work studies the null-controllability of a class of abstract parabolic equations. The main contribution in the general case consists in giving a short proof of an abstract version of a sufficient condition for null-controllability which has been proposed by Lebeau and Robbiano. We do not assume that the control operator is admissible. Moreover, we give estimates of the control cost. In the special case of the heat equation in rectangular domains, we provide an alternative way to check the Lebeau-Robbiano spectral condition. We then show that the sophisticated Carleman and interpolation inequalities used in previous literature may be replaced by a simple result of Turán. In this case, we provide explicit values for the constants involved in the above mentioned spectral condition. As far as we are aware, this is the first proof of the null-controllability of the heat equation with arbitrary control domain in a $n$-dimensional open set which avoids Carleman estimates.
\end{abstract}

Mathematics Subject Classification. 93C25, 93B07, 93C20.

Received August 29, 2009. Revised April 21, 2010.

Published online August 23, 2010.

\section{INTRODUCTION AND MAIN RESULTS}

This work is concerned with the study of null-controllability for a class of infinite dimensional systems described by abstract parabolic equations of the form

$$
\dot{w}=A w+B u, w(0)=z
$$

where $A$ is a negative operator in a Hilbert space $X$ and, say, the operator $B$ maps the input space $U$ (also a Hilbert space) into $X$. As discussed below, the range of $B$ can in fact be a space which is larger than $X$ : we say in this case that the input operator in unbounded. More precise assumptions on $A$ and $B$ will be made later.

In the special case when $A$ is the Dirichlet Laplacian in an open bounded set $\Omega \subset \mathbb{R}^{n}$ and $B$ corresponds to a control distributed in a subset of $\Omega$, i.e., for the standard diffusion equation, the null-controllability properties are well understood. For early contributions, in which $A$ is the Dirichlet Laplacian in one space dimension, we refer to Fattorini and Russell [1,2].

When $A$ is the Dirichlet Laplacian in $\Omega \subset \mathbb{R}^{n}$, where $n \geqslant 2$, and the control $u$ is supported on an arbitrary open subset of $\Omega$, the null-controllability result has been independently established by Lebeau and Robbiano [4], and by Fursikov and Imanuvilov [3].

\footnotetext{
Keywords and phrases. Heat equation, controllability, spectral condition, Turán's method.

1 Institut Élie Cartan, Nancy Université/CNRS/INRIA, BP 70239, 54506 Vandœuvre-lès-Nancy Cedex, France.

gerald.tenenbaum@iecn.u-nancy.fr; tucsnak@iecn.u-nancy.fr
} 
More recently, Micu and Zuazua in [6] (in one space dimension) and Miller [7] (in several space dimensions) obtained a null-controllability result when $A=-A_{0}^{\eta}$, where $\eta>\frac{1}{2}$ and $-A_{0}$ is the Dirichlet Laplacian. The main tool brought up in [7] is an abstract sufficient condition for null-controllability, inspired by the method introduced in [4] - see also Lebeau and Zuazua [5]. Note that the spectral condition in [7] is stated for a $A=-A_{0}^{\eta}$, with a general positive operator $A_{0}$ (not necessarily with discrete spectrum) and that it furnishes estimates of the control cost. The work of Seidman [10] contains a new version of the Lebeau-Robbiano method which allowed, at least for $\eta=1$ and $B \in \mathscr{L}(U, X)$, the improvement of the estimates from [7]. The constants in the control cost have been further improved in [8], where a more direct methodology has been proposed.

In this work, our initial aim consists, in an abstract setting, in relaxing the assumptions on the control operator $B$ with respect to the existing literature, together with simplifying some proofs and the form of the constants involved in the control cost estimates. In this context, without assuming any admissibility property of the control operator $B$, we show, without any duality argument, that the observability assumption of finite combinations of eigenvectors of the pair $\left(A^{*}, B^{*}\right)$ implies its final-state observability. Note that a proof of this implication has also been, independently and almost simultaneously, provided in [8] under the assumption that $B$ is an admissible observation operator for the semigroup generated by $A$.

Our second purpose is, in the case when $A=-A_{0}^{\eta}$, where $A_{0}$ is the Dirichlet Laplacian in a rectangular domain $\Omega$ and $\eta>1 / 2$, to provide an alternative way to check the Lebeau-Robbiano spectral condition. We show, in this particular case, that the sophisticated Carleman and interpolation inequalities used in [4] may be replaced by a simple result of Turán [12]. A novelty brought in by our approach is that, in the case of rectangular domains, we provide explicit values for the constants involved in the above mentioned spectral condition. Moreover, as far as we are aware, this is the first proof of the null-controllability of the heat equation with arbitrary control domain in a $n$-dimensional open set which avoids Carleman estimates.

In order to precisely state our results, we need further notation. Let $X$ be a Hilbert space, called state space, which will be identified with its dual. The inner product in $X$ is denoted simply by $\langle\cdot, \cdot\rangle\rangle$ and the subordinated norm by $\|\cdot\|$. Let $A: \mathscr{D}(A) \rightarrow X$ be a a self-adjoint operator (possibly unbounded) on $X$ such that

$$
\langle A z, z\rangle \leqslant 0 \quad(z \in \mathscr{D}(A)) .
$$

Such an operator will be briefly called a negative operator. We also assume that $A$ is diagonalizable with an orthonormal basis of eigenvectors $\left\{\varphi_{k}\right\}_{k \geqslant 0}$ and corresponding family of eigenvalues $\left\{-\lambda_{k}\right\}_{k \geqslant 0}$, where the sequence $\left(\lambda_{k}\right)$ is positive, non decreasing and satisfies $\lambda_{k} \rightarrow \infty$. According to classical results, this holds, in particular, if $A$ has compact resolvents.

With the above assumptions on $A$, we have

$$
A z=-\sum_{k \geqslant 0} \lambda_{k}\left\langle z, \varphi_{k}\right\rangle \varphi_{k} \quad(z \in \mathscr{D}(A)),
$$

so $A$ generates a contraction semigroup $\mathbb{T}$ on $X$ satisfying

$$
\mathbb{T}_{t} z=\sum_{k \geqslant 0} \mathrm{e}^{-\lambda_{k} t}\left\langle z, \varphi_{k}\right\rangle \varphi_{k} \quad(t \geqslant 0, z \in X) .
$$

Moreover, the sets

$$
X_{\beta}:=\left\{z \in X: \sum_{k \geqslant 0}\left(1+\lambda_{k}^{2}\right)^{\beta}\left|\left\langle z, \varphi_{k}\right\rangle\right|^{2}<\infty\right\} \quad(\beta>0)
$$

endowed with the inner product

$$
\langle y, z\rangle_{\beta}=\sum_{k \geqslant 0}\left(1+\lambda_{k}^{2}\right)^{\beta}\left\langle y, \varphi_{k}\right\rangle \overline{\left\langle z, \varphi_{k}\right\rangle} \quad\left(z, y \in X_{\beta}\right)
$$

are Hilbert spaces. The scale $\left\{X_{\beta}\right\}_{\beta \geqslant 0}$ of Hilbert spaces can be extended to a scale $\left\{X_{\beta}\right\}_{\beta \in \mathbb{R}}$ by defining, for every $\beta<0, X_{\beta}$ as the completion of $X$ with respect to the norm associated to the inner product (1.5). 
Alternatively, $X_{-\beta}$ may be defined, for $\beta>0$, as the dual of $X_{\beta}$ with respect to the pivot space $X$. For every $\beta>0$, formulas (1.2) and (1.3), with $\langle\cdot, \cdot\rangle$ standing this time for the duality between $X_{-\beta}$ and $X_{\beta}$, provide canonical extensions for the operator $A$ and the semigroup $\mathbb{T}$ to, respectively, a negative operator and a contraction semigroup on $X_{-\beta}$-indeed, the corresponding series converge in $X_{-\beta}$. These extensions will be still denoted by $A$ and $\mathbb{T}$. Note that, for every $\beta \in \mathbb{R}$, the family $\left\{\left(1+\lambda_{k}^{2}\right)^{\beta / 2} \varphi_{k}\right\}_{k \geqslant 0}$ is an orthonormal basis in $X_{\beta}$.

Remark 1.1. The operator $A$ is not explicitly necessary to construct the Hilbert spaces scale $\left\{X_{\beta}\right\}_{\beta \in \mathbb{R}}$. Indeed, one only needs to consider, formally, the Hilbert space $X$, a non decreasing, non negative sequence $\left\{\lambda_{n}\right\}_{n} \geqslant 0$, with $\lambda_{n} \rightarrow \infty$, and an orthonormal basis $\left\{\varphi_{n}\right\}_{n \geqslant 0}$ in $X$.

Let $U$ be another Hilbert space, called input space, also identified with its dual. Let $\beta \geqslant 0$ and let $B \in$ $\mathscr{L}\left(U, X_{-\beta}\right)$ be an input operator. With the above notation, the solution $w=w_{z}$ of (1.1) is defined by

$$
w_{z}(t)=\mathbb{T}_{t} z+\int_{0}^{t} \mathbb{T}_{t-s} B u(s) \mathrm{d} s \quad\left(t \geqslant 0, z \in X, u \in L^{2}([0, \infty[, U)) .\right.
$$

Note that, in general, $w_{z} \notin C\left(\left[0, \infty[, X)\right.\right.$, so that (1.6) should be understood in $C\left(\left[0, \infty\left[, X_{-\beta}\right)\right.\right.$. The system (1.1) is said to be null-controllable in time $T>0$ if, for every $z \in X$, there exists $u \in L^{2}([0, T], U)$ such that $w_{z}(T)=0$, i.e., if, for every $z \in X$, the set

$$
\mathscr{C}_{T, z}:=\left\{u \in L^{2}([0, T], U): w_{z}(T)=0\right\}
$$

is non empty. The quantity

$$
C_{T}:=\sup _{\|z\|=1} \inf _{u \in \mathscr{C}_{T, z}}\|u\|_{L^{2}([0, T], U)}
$$

is then called the control cost.

Note that, since $X, U$ are identified with their duals, we have $B^{*} \in \mathscr{L}\left(X_{\beta}, U\right)$.

Below and in the sequel, we freely use Vinogradov's «-notation. Thus a formula of the type $f(x) \ll g(x)$ $(x \in X)$ indicates that, for all $x$ in the set $X$, the inequality $|f(x)| \leq C|g(x)|$ holds with a suitable constant $C>0$, which may depend on certain implicit parameters. In this last case, the dependence may, or may not, be indicated by annotating the symbol with appropriate subscripts.

We now state our first main result.

Theorem 1.2. Let $A: \mathscr{D}(A) \rightarrow X$ be a negative operator and let $B \in \mathscr{L}\left(U, X_{-\beta}\right)$ for some $\beta \geqslant 0$. Assume that $A$ is diagonalizable, that $\left\{\varphi_{k}\right\}_{k=0}^{\infty}$ is an orthonormal basis of eigenvectors with corresponding non increasing sequence of eigenvalues $\left\{-\lambda_{k}\right\}_{k=0}^{\infty}$ such that $\lim \lambda_{k}=\infty$. Assume furthermore that there exists $\left.\gamma \in\right] 0,1[$ such that, for suitable positive constants $d_{0}, d_{1}$, we have

$$
\left(\sum_{\lambda_{k}^{\gamma} \leqslant \mu}\left|a_{k}\right|^{2}\right)^{1 / 2} \leqslant d_{0} \mathrm{e}^{d_{1} \mu}\left\|\sum_{\lambda_{k}^{\gamma} \leqslant \mu} a_{k} B^{*} \varphi_{k}\right\|_{U} \quad\left(\left\{a_{k}\right\}_{k \geqslant 0} \in \ell^{2}(\mathbb{C}), \mu>1\right) .
$$

Then the system (1.1) is null-controllable in any time $T>0$. Moreover, given $c>h^{g h} g^{-g^{2}} d_{1}^{h}$, where $g:=$ $\gamma /(1-\gamma), h:=g+1=1 /(1-\gamma)$, the control cost satisfies

$$
C_{T} \ll T^{-1 / 2} \mathrm{e}^{c / T^{g}} \quad(T>0),
$$

where the implicit constant depends only on $d_{0}, d_{1}, c, \beta, \gamma$ and $\|B\|_{\mathscr{L}\left(U, X_{-\beta}\right)}$.

Remark 1.3. For small $T$, the condition on $c$ in the above theorem coincides with the one obtained in [8], where a larger family of generators $A$ is considered - in particular $A$ is not supposed to be negative or with discrete 
spectrum, so that assumption (1.7) is stated in a more general form. Unlike in [8], we do not assume in Theorem 1.2 that $B$ is an admissible control operator for the semigroup generated by $A$ - see, for instance [11], Section 4.2, for the definition of this admissibility concept.

Remark 1.4. From (1.8), it follows that there exists a constant $K>0$ such that $C_{T} \leqslant K / \sqrt{T}$ for large $T$. This decay rate is sharp if $\lambda_{0}=0$ since, if $u_{0}$ is a control steering the initial state $\varphi_{0}$ to zero in time $T$, then

$$
\int_{0}^{T}\left\langle u_{0}(t), \varphi_{0}\right\rangle \mathrm{d} t=-1
$$

so that the Cauchy-Schwarz inequality clearly yields $\left\|u_{0}\right\|_{L^{2}([0, T], U)} \geqslant 1 / \sqrt{T}$. However, if $\lambda_{0}>0$, then $C_{T}$ decays exponentially as $T \rightarrow \infty$. Indeed, we can then select $u:=0$ on $[0, T / 2]$ and then steer $z$ to 0 at time $T$ by applying, on $[T / 2, T]$, a control uniformly bounded for $T>0$ : that this is possible follows from Theorem 1.2 .

A typical range of application for Theorem 1.2 is provided by diffusion equations (possibly fractional) in an open bounded set $\Omega \subset \mathbb{R}^{n}$, with control acting in an arbitrary open set $\mathcal{O} \subset \Omega$. The major difficulty is then to prove (1.7). This problem is solved in [4] when $A:=-A_{0}^{\eta}, \eta>\frac{1}{2}, \gamma:=1 /(2 \eta)$, and $-A_{0}$ is the Dirichlet Laplacian on $\Omega$, provided that $\partial \Omega$ is of class $C^{\infty}$. The corresponding proof uses deep Carleman estimates and interpolation inequalities. In Section 3, we tackle the case of rectangular $\Omega$ by a different and fully elementary method. More precisely, we prove the following result.

Theorem 1.5. Let $m \in \mathbb{N}^{*}, \Omega \subset \mathbb{R}^{m}$ be a rectangular domain, $X:=L^{2}(\Omega)$, let $A:=-A_{0}^{\eta}$, where $-A_{0}$ is the Dirichlet or the Neumann Laplacian in $\Omega$ and $\eta>1 / 2$. Set $U:=L^{2}(\mathcal{O})$ and define $B \in \mathscr{L}(U, X)$ by the formula

$$
B v:=v \mathbb{1}_{\mathcal{O}} \quad(v \in U),
$$

where $\mathbb{1}_{\mathcal{O}}$ is the characteristic function of a non empty open subset $\mathcal{O}$ of $\Omega$. Then assumption (1.7) holds with $\gamma:=1 /(2 \eta)$.

In particular, if $\Omega=[0, \pi]^{m}$, then, given any $\varepsilon>0$, we may select

$$
d_{1}:=3(1+\varepsilon) m \log (4 \pi \mathrm{e})-3(1+\varepsilon) \log \left|\mathcal{O}^{*}\right|
$$

where $\mathcal{O}^{*}$ is any hyper-rectangle included in $\mathcal{O}$, and

$$
d_{0}:=2^{-m / 4} \pi^{m / 2}\left(\frac{1}{\mathrm{e} \varepsilon \log (4 \mathrm{e})}\right)^{3 m / 4} .
$$

By combining Theorem 1.5 (for a rectangular $\Omega$ ) or the results in [4] (for the case in which $\partial \Omega$ is $C^{\infty}$ ) with Theorem 1.2 with $\gamma:=1 /(2 \eta)$, we obtain the following statement.

Corollary 1.6. Let $m \in \mathbb{N}^{*}$, let $\Omega \subset \mathbb{R}^{m}$ be a rectangular domain or a bounded domain with $C^{\infty}$ boundary and assume the spaces $X, U$ and the operators $A, B$ verify the hypotheses of Theorem 1.5. Then the system (1.1) is null-controllable in any time $T>0$. Moreover, there exists a constant $K>0$ such that the control cost satisfies

$$
C_{T} \ll T^{-1 / 2} \exp \left\{K T^{-1 /(2 \eta-1)}\right\} \quad(T>0),
$$

where the implicit constant depends only on $\Omega, \mathcal{O}$ and $K$. Moreover, in the case $\Omega=[0, \pi]^{m}$, the constant $K$ can be chosen to be any number satisfying the condition

$$
K>(2 \eta)^{2 \eta /(2 \eta-1)^{2}}(2 \eta-1)^{-1 /(2 \eta-1)} d_{1}^{2 \eta /(2 \eta-1)}
$$

where $d_{1}$ is the constant defined in (1.9). 


\section{Study of A MOMEnT PROBlem}

This section is devoted to set up the main argument of the proof of Theorem 1.2. Given $T>0$, a sequence $\left\{\psi_{k}\right\}_{k \geqslant 0}$ in a Hilbert space $U$ and a non decreasing real sequence $\left\{\lambda_{k}\right\}_{k \geqslant 0}$, we are interested in the solvability of the moment problem which consists in finding, for every sequence $\left\{a_{k}\right\}_{k \geqslant 0}$, a function $\Theta \in L^{2}([0, T], U)$ such that

$$
a_{n} \mathrm{e}^{-\lambda_{n} T}+\int_{0}^{T} \mathrm{e}^{-\lambda_{n} t}\left\langle\Theta(t), \psi_{n}\right\rangle_{U} \mathrm{~d} t=0 \quad(n \geqslant 0) .
$$

Appealing to a simplification of the methodology proposed in [4] and developed in $[7,8,10]$ we give below sufficient conditions for the solvability of this moment problem and provide estimates for the solution $\Theta$.

We start by recalling a classical result from Hilbert space theory. For the sake of completeness, we provide a short proof, inspired from [11], Section 12.1 .

Lemma 2.1. Suppose that $W, Y, Z$ are Hilbert spaces, $\mathscr{F} \in \mathscr{L}(W, Z)$ and that $\mathscr{G} \in \mathscr{L}(Y, Z)$. Then the following statements are equivalent:

(i) there exists $c>0$ such that

$$
\left\|\mathscr{F}^{*} z\right\|_{W} \leqslant c\left\|\mathscr{G}^{*} z\right\|_{Y} \quad(z \in Z)
$$

(ii) there exists $\mathscr{H} \in \mathscr{L}(W, Y)$ such that $\mathscr{G} \mathscr{H}=\mathscr{F}$ and $\|\mathscr{H}\|_{\mathscr{L}(W, Y)} \leqslant c$.

Proof. That (ii) implies (i) readily follows from the facts that $\mathscr{H}^{*} \mathscr{G}^{*}=\mathscr{F}^{*}$ and $\left\|\mathscr{H}^{*}\right\|_{\mathscr{L}(Y, W)}=\|\mathscr{H}\|_{\mathscr{L}(W, Y)}$. It remains to show that (i) implies (ii). Assume (i) holds. We may define a mapping $\mathscr{K}: \operatorname{Ran} \mathscr{G}^{*} \rightarrow \operatorname{Ran} \mathscr{F}^{*}$ by the formula $\mathscr{K}\left(\mathscr{G}^{*} z\right)=\mathscr{F}^{*} z$ for all $z \in Z$. Indeed, $\mathscr{K}$ is well defined since $\mathscr{G}^{*} z_{1}=\mathscr{G}^{*} z_{2}$ implies

$$
\left\|\mathscr{F}^{*}\left(z_{1}-z_{2}\right)\right\|_{W} \leq c\left\|\mathscr{G}^{*}\left(z_{1}-z_{2}\right)\right\|_{Y}=0
$$

and so $\mathscr{F}^{*} z_{1}=\mathscr{F}^{*} z_{2}$. Moreover, condition (i) can be rewritten as

$$
\left\|\mathscr{K}\left(\mathscr{G}^{*} z\right)\right\|_{W} \leqslant c\left\|\mathscr{G}^{*} z\right\|_{Y} \quad(z \in Z)
$$

Hence, $\mathscr{K}$ has a unique continuous extension to the closure $\overline{\operatorname{Ran} \mathscr{G}^{*}}$ such that

$$
\|\mathscr{K} y\|_{W} \leqslant c\|y\|_{Y} \quad\left(y \in \overline{\operatorname{Ran} \mathscr{G}^{*}}\right) .
$$

Next, we extend $\mathscr{K}$ to $Y$ by setting $\mathscr{K} y=0$ for $y \in\left(\operatorname{Ran} \mathscr{G}^{*}\right)^{\perp}$. We still have $\mathscr{K} \mathscr{G}^{*} z=\mathscr{F}^{*} z$ for every $z \in Z$ and if $y=y_{1}+y_{2} \in Y$ with $y_{1} \in \overline{\operatorname{Ran} \mathscr{G}^{*}}$ and $y_{2} \in\left(\operatorname{Ran} \mathscr{G}^{*}\right)^{\perp}$, we infer from (2.1) that

$$
\|\mathscr{K} y\|_{W}=\left\|\mathscr{K} y_{1}\right\|_{W} \leqslant c\left\|y_{1}\right\|_{Y} \leqslant c\|y\|_{Y} \quad(y \in Y),
$$

so that $\|\mathscr{K}\|_{\mathscr{L}(Y, W)} \leqslant c$. Consequently, the operator $\mathscr{H}=\mathscr{K}^{*}$ satisfies the required conditions.

The main result of this section is Proposition 2.3 below. This statement rests upon a double set of hypotheses concerning the sequence $\left\{\psi_{n}\right\}_{n=0}^{\infty}$ of vectors of $U$ and the non decreasing sequence of non-negative real numbers $\left\{\lambda_{n}\right\}_{n=0}^{\infty}$, namely

$$
(H 1)\left\{\begin{array}{l}
\text { (i) } \lambda_{n} \geqslant C n^{\alpha} \text { for all } n \in \mathbb{N} \text { and suitable constants } \alpha>0, C>0, \\
\text { (ii) }\left\|\psi_{n}\right\|_{U} \leqslant M\left(1+\lambda_{n}\right)^{\beta}(n \geqslant 0) \text { for some } M>0, \beta>0
\end{array}\right.
$$

and

$$
(H 2)\left\{\begin{aligned}
\text { (i) } & \lim _{n \rightarrow \infty} \lambda_{n}=\infty, \\
\text { (ii) } \quad & \psi_{n}=B^{*} \varphi_{n}(n \geqslant 0) \text { where }\left\{\varphi_{n}\right\}_{n=0}^{\infty} \text { is an orthonormal basis of } X \\
& \text { and } B \in \mathscr{L}\left(U, X_{-\beta}\right) \text { for some } \beta>0,
\end{aligned}\right.
$$


where the scale of Hilbert spaces $\left\{X_{\beta}\right\}_{\beta \in \mathbb{R}}$ is formally constructed from the sequences $\left(\lambda_{n}\right)$ and $\left(\varphi_{n}\right)$ as described in Remark 1.1.

We exploit these assumptions in the following way (recall from the previous section the notation $\ll$ for the Vinogradov symbol).

Lemma 2.2. Let $\left\{\psi_{n}\right\}_{n=0}^{\infty}$ be a sequence of vectors of $U$ and $\left\{\lambda_{n}\right\}_{n=0}^{\infty}$ be a non decreasing sequence of non-negative real numbers such that one of the hypotheses $(H 1)$ or $(H 2)$ is satisfied. Let $\left\{a_{n}\right\}_{n=0}^{\infty} \in \ell^{2}(\mathbb{C})$, $\lambda>0$ and denote

$$
\begin{aligned}
& F(t):=\sum_{n \geqslant 0}\left|a_{n}\right|^{2} \mathrm{e}^{-2 \lambda_{n} t}, \quad F_{\lambda}(t):=\sum_{\lambda_{n} \leqslant \lambda}\left|a_{n}\right|^{2} \mathrm{e}^{-2 \lambda_{n} t}, \quad F_{\lambda}^{\perp}(t):=F(t)-F_{\lambda}(t), \\
& G_{\lambda}(t):=\left\|\sum_{\lambda_{n} \leqslant \lambda} a_{n} \mathrm{e}^{-\lambda_{n} t} \psi_{n}\right\|_{U}^{2}, \quad G_{\lambda}^{\perp}(t):=\left\|\sum_{\lambda_{n}>\lambda} a_{n} \mathrm{e}^{-\lambda_{n} t} \psi_{n}\right\|_{U}^{2} .
\end{aligned}
$$

Then, for all positive real numbers $\varepsilon, g$, $\vartheta$ and $\eta$ such that $\vartheta+\eta+\varepsilon=1$, we have

$$
F_{\lambda}^{\perp}(t)+G_{\lambda}^{\perp}(t) \ll \mathrm{e}^{-2 \vartheta \lambda t+\varepsilon / t^{g}} F(\eta t) \quad(t>0) .
$$

The implicit constant may depend on $\alpha, \beta, C, \varepsilon, g, M$ in the first case, and on $B, \beta, \varepsilon, g$ in the second.

Proof. It clearly suffices to prove the upper bound for $G_{\lambda}^{\perp}$. Assume first that $(H 1)$ holds. Then, by the Cauchy-Schwarz inequality,

$$
\begin{aligned}
G_{\lambda}^{\perp}(t) & \leqslant M^{2} \sum_{\lambda_{n}>\lambda} \mathrm{e}^{-3 \varepsilon \lambda_{n} t / 2} \sum_{\lambda_{n}>\lambda}\left|a_{n}\right|^{2} \mathrm{e}^{-(2-3 \varepsilon / 2) \lambda_{n} t}\left(1+\lambda_{n}\right)^{2 \beta} \\
& \ll_{\beta, \varepsilon} M^{2}\left(1+t^{-2 \beta}\right) \sum_{n \geqslant 0} \mathrm{e}^{-3 C \varepsilon n^{\alpha} t / 2} \sum_{\lambda_{n}>\lambda}\left|a_{n}\right|^{2} \mathrm{e}^{-(2-2 \varepsilon) \lambda_{n} t} .
\end{aligned}
$$

Since

we obtain

$$
\sum_{n \geqslant 0} \mathrm{e}^{-3 C \varepsilon n^{\alpha} / 2} \leqslant 1+\int_{0}^{\infty} \mathrm{e}^{-3 C \varepsilon x^{\alpha} / 2} \mathrm{~d} x=1+\frac{\Gamma(1+1 / \alpha)}{(3 C \varepsilon t / 2)^{1 / \alpha}}
$$

$$
G_{\lambda}^{\perp}(t) \ll\left(1+t^{-1 / \alpha}\right)\left(1+t^{-2 \beta}\right) \mathrm{e}^{-2 \vartheta \lambda t} F(\eta t) \ll \mathrm{e}^{-2 \vartheta \lambda t+\varepsilon / t^{g}} F(\eta t) .
$$

Assume now that $(H 2)$ holds. Then, Parseval's formula implies that

$$
\begin{aligned}
G_{\lambda}^{\perp}(t) & =\left\|B^{*} \sum_{\lambda_{n}>\lambda} a_{n} \mathrm{e}^{-\lambda_{n} t} \varphi_{n}\right\|_{U}^{2} \leqslant\|B\|_{\mathscr{L}\left(U, X_{-\beta}\right)}^{2} \sum_{\lambda_{n}>\lambda}\left|a_{n}\right|^{2} \mathrm{e}^{-2 \lambda_{n} t}\left(1+\lambda_{n}^{2}\right)^{\beta} \\
& \ll\|B\|_{\mathscr{L}\left(U, X_{-\beta}\right)}^{2}\left(1+t^{-2 \beta}\right) \mathrm{e}^{-2 \lambda \vartheta t} F(\eta t),
\end{aligned}
$$

where, in the last stage, we used the bound

$$
\left(1+\lambda_{n}^{2}\right)^{\beta} \mathrm{e}^{-2 \lambda_{n}(\vartheta+\eta+\varepsilon) t} \ll\left(1+t^{-2 \beta}\right) \mathrm{e}^{-2 \vartheta \lambda t-2 \eta \lambda_{n} t} \quad\left(\lambda_{n}>\lambda, t>0\right) .
$$

This readily implies the required conclusion.

We are now in a position to state the main result in this section.

Proposition 2.3. With the notation and assumptions of Lemma 2.2, put

$$
G(t):=\left\|\sum_{n \geqslant 0} a_{n} \mathrm{e}^{-\lambda_{n} t} \psi_{n}\right\|^{2} \quad(t>0),
$$


and assume furthermore that there exists $\gamma \in] 0,1\left[\right.$ such that, for suitable positive constants $d_{0}$, $d_{1}$, we have

$$
\left(\sum_{\lambda_{k}^{\gamma} \leqslant \mu}\left|a_{k}\right|^{2}\right)^{1 / 2} \leqslant d_{0} \mathrm{e}^{d_{1} \mu}\left\|\sum_{\lambda_{k}^{\gamma} \leqslant \mu} a_{k} \psi_{k}\right\|_{U} \quad\left(\left\{a_{k}\right\}_{k \geqslant 0} \in \ell^{2}(\mathbb{C}), \mu>1\right) .
$$

Let $g:=\gamma /(1-\gamma), h:=1 /(1-\gamma)$. Given $D>2 h^{g h} g^{-g^{2}} d_{1}^{h}$, we have

$$
F(T) \ll \frac{\mathrm{e}^{D / T^{g}}}{T} \int_{0}^{T} G(t) \mathrm{d} t \quad(T>0) .
$$

The implicit constant above may depend upon $\gamma, d_{0}, d_{1}, D$, and on $\alpha, \beta, C, M$ if hypothesis (H1) holds, or B, $\beta$ if assumption (H2) is satisfied.

Proof. Let $r>1$ and $\left\{a_{k}\right\}_{k \geqslant 0} \in \ell^{2}(\mathbb{C})$. By (2.4), we have

$$
F_{\lambda}(t) \leqslant d_{0}^{2} \mathrm{e}^{2 d_{1} \lambda^{\gamma}} G_{\lambda}(t) \quad(\lambda>0, T / r \leqslant t \leqslant T) .
$$

Integrating this with respect to $t$ and using the trivial bound

$$
\frac{(r-1) T}{r} \mathrm{e}^{-2 \lambda_{k} T} \leqslant \int_{T / r}^{T} \mathrm{e}^{-2 \lambda_{k} t} \mathrm{~d} t \quad(k \in \mathbb{N}),
$$

we obtain

$$
F_{\lambda}(T) \leqslant \frac{K_{1}}{T} \mathrm{e}^{K_{2} \lambda^{\gamma}} \int_{T / r}^{T} G_{\lambda}(t) \mathrm{d} t \quad(T>0)
$$

with

$$
K_{1}:=r d_{0}^{2} /(r-1), \quad K_{2}:=2 d_{1} .
$$

Let $\varepsilon>0$ and let $\vartheta, \sigma>0$ be such that $\varepsilon=1-\vartheta-r \sigma$. Recall that $g:=\gamma /(1-\gamma)$ and $h:=1 /(1-\gamma)$. Using Lemma 2.2 and (2.6), it is not difficult to check that

$$
F(T) \ll \frac{\mathrm{e}^{K_{2} \lambda^{\gamma}+\varepsilon / T^{g}}}{T} \int_{T / r}^{T} G(t) \mathrm{d} t+\mathrm{e}^{K_{2} \lambda^{\gamma}-2 \vartheta \lambda T+\varepsilon / T^{g}} F(\sigma T),
$$

where the implicit constant may depend upon $K_{1}$ and all parameters indicated in the statement of Lemma 2.2. Given $\nu>0$, we select $\lambda:=\left\{K_{2} \nu / \vartheta \sigma T\right\}^{h}$, so that

$$
K_{2} \lambda^{\gamma}-2 \vartheta \lambda T=\frac{K_{2}^{h} \nu^{g}}{(\vartheta \sigma T)^{g}}\left\{1-\frac{2 \nu}{\sigma}\right\}=-\frac{K_{2}^{h} \nu^{g}(2 \nu-\sigma)}{\vartheta^{g} \sigma^{h} T^{g}} .
$$

Writing

equation (2.7) becomes

$$
D_{0}:=\frac{K_{2}^{h} \nu^{g}}{\vartheta^{g} \sigma^{g}}, \quad E_{0}:=\frac{K_{2}^{h} \nu^{g}(2 \nu-\sigma)}{\vartheta^{g} \sigma^{h}}
$$

$$
F(T) \leqslant \frac{K}{T} \mathrm{e}^{D / T^{g}} \int_{T / r}^{T} G(t) \mathrm{d} t+K \mathrm{e}^{-E / T^{g}} F(\sigma T),
$$

where $K$ is a suitable constant and

$$
D:=D_{0}+\varepsilon, \quad E:=E_{0}-\varepsilon
$$

Selecting $\nu$ so large that

$$
\nu>\frac{1}{2 \sigma^{g-1}}+\frac{\varepsilon \sigma \vartheta^{g}}{2 K_{2}^{h} \nu^{g}},
$$


we have

$$
E>D\left(1 / \sigma^{g}-1\right)
$$

Applying (2.8) to $F(\sigma T)$ instead of $F(T)$, we thus arrive at

$$
F(T) \leqslant \frac{K}{T} \mathrm{e}^{D / T^{g}} \int_{T / r}^{T} G(t) \mathrm{d} t+\frac{K^{2}}{\sigma T} \mathrm{e}^{D_{1} / T^{g}} \int_{\sigma T / r}^{\sigma T} G(t) \mathrm{d} t+K^{2} \mathrm{e}^{-E_{1} / T^{g}} F\left(\sigma^{2} T\right)
$$

where

$$
D_{1}:=D / \sigma^{g}-E, \quad E_{1}:=E\left(1+\sigma^{g}\right) / \sigma^{g}
$$

After $N$ iterations, we thus get

$$
\begin{aligned}
F(T) \leqslant & \frac{K}{T} \mathrm{e}^{D / T^{g}} \int_{T / r}^{T} G(t) \mathrm{d} t+\sum_{1 \leqslant j \leqslant N} \frac{K^{j+1}}{\sigma^{j} T} \mathrm{e}^{D_{j} / T^{g}} \int_{\sigma^{j} T / r}^{\sigma^{j} T} G(t) \mathrm{d} t \\
& +K^{N+1} \mathrm{e}^{-E_{N} / T^{g}} F\left(\sigma^{N+1} T\right),
\end{aligned}
$$

with

$$
D_{j}:=\frac{D-E\left(\sigma^{g}+\ldots+\sigma^{g j}\right)}{\sigma^{g j}}=\frac{1}{\sigma^{g j}}\left\{D-\frac{E\left(1-\sigma^{j g}\right)}{1 / \sigma^{g}-1}\right\}, \quad E_{N}:=\frac{E\left(1+\ldots+\sigma^{g N}\right)}{\sigma^{g N}} .
$$

Observe that $\sup _{t \geqslant 0} F(t) \leqslant\|a\|_{\ell^{2}(\mathbb{C})}$, so that the last term in (2.11) tends to 0 as $N \rightarrow \infty$. Moreover, we infer from (2.10) that $D_{j}<D$ for all $j$ and that $D_{j}$ tends exponentially to $-\infty$ as $j \rightarrow \infty$. Therefore, under the above restrictions on the various parameters, we obtain

$$
\sum_{j \geqslant 1} \frac{K^{j+1}}{\sigma^{j} T} \mathrm{e}^{D_{j} / T^{g}} \ll \frac{\mathrm{e}^{D / T^{g}}}{T}
$$

if, say, $T \leqslant 1$. The same result holds for $T>1$ provided we increase, if needed, the value of $\nu$ to ensure that $D_{1}=-L T^{g}$ where $L$ is a constant possibly depending on all parameters except $T$ and such that $L>2 K / \sigma$ : then, we have $\mathrm{e}^{D / T^{g}} \ll 1$ and the alteration of $D$ is actually irrelevant.

Letting $N \rightarrow \infty$ in (2.11), we finally obtain

$$
F(T) \ll \frac{\mathrm{e}^{D / T^{g}}}{T} \int_{0}^{T} G(t) \mathrm{d} t,
$$

where the implicit constant is restricted as in the statement.

This plainly implies the stated result when $T>1$ since, as noted above, the exponential factor is bounded with our choice for $\nu$. When $T \leqslant 1$, we may select any $\vartheta<1 / h, \sigma<g / h, \nu>2^{g-2}$ provided $r$ is sufficiently close to 1 and $\varepsilon$ is small enough. Then, $D$ can be taken as any number strictly exceeding $2^{-g} h^{g h} g^{-g^{2}} K_{2}^{h}$. Since $K_{2}=2 d_{1}$, we indeed get that (2.5) holds whenever $D>2 h^{g h} g^{-g^{2}} d_{1}^{h}$.

Proposition 2.4. With the notation and assumptions of Proposition 2.3, for every $T>0$ there exists a bounded operator $H_{T} \in \mathscr{L}\left(\ell^{2}(\mathbb{C}), L^{2}([0, T], U)\right)$ such that, for every $\left\{a_{n}\right\}_{n \geqslant 0} \in \ell^{2}(\mathbb{C})$, we have

$$
a_{n} \mathrm{e}^{-\lambda_{n} T}+\int_{0}^{T} \mathrm{e}^{-\lambda_{n} t}\left\langle H_{T} a(t), \psi_{n}\right\rangle_{U} \mathrm{~d} t=0 \quad(n \geqslant 0)
$$

Moreover, for every $c>h^{g h} g^{-g^{2}} d_{1}^{h}$, we have

$$
\left\|H_{T}\right\| \ll \frac{\mathrm{e}^{c / T^{g}}}{\sqrt{T}} \quad(T>0) .
$$


The implicit constant above may depend upon $\gamma, d_{0}, d_{1}, D$ and on $\alpha, \beta, C, M$ if hypothesis (H1) holds, or B, $\beta$ if assumption $(\mathrm{H} 2)$ is satisfied.

Proof. Write $Y:=L^{2}([0, T], U), Z:=\ell^{2}(\mathbb{C})$. Here $Y$ and $Z$ are endowed with the canonical scalar products

$$
\langle u, v\rangle_{Y}:=\int_{0}^{T}\langle u(t), v(t)\rangle_{U} \mathrm{~d} t, \quad\langle a, b\rangle_{Z}:=\sum_{n \geqslant 0} a_{n} \overline{b_{n}}
$$

Given $\varepsilon>0$, we consider $\mathscr{G}_{\varepsilon} \in \mathscr{L}(Y, Z)$ defined by

$$
\left(\mathscr{G}_{\varepsilon} u\right)_{n}:=\left\langle u, \mathrm{e}^{-\lambda_{n}(t+\varepsilon)} \psi_{n}\right\rangle_{Y} \quad(n \geqslant 0)
$$

Then for any $a \in Z$, we have

$$
\left\langle\mathscr{G}_{\varepsilon} u, a\right\rangle_{Z}=\sum_{n \geqslant 0} \overline{a_{n}}\left\langle u, \mathrm{e}^{-\lambda_{n}(t+\varepsilon)} \psi_{n}\right\rangle_{Y}=\left\langle u, \sum_{n \geqslant 0} a_{n} \mathrm{e}^{-\lambda_{n}(t+\varepsilon)} \psi_{n}\right\rangle_{Y}
$$

Therefore,

$$
\mathscr{G}_{\varepsilon}^{*} a=\sum_{n \geqslant 0} a_{n} \mathrm{e}^{-\lambda_{n}(t+\varepsilon)} \psi_{n} \quad\left(a \in \ell^{2}(\mathbb{C})\right) .
$$

Consider also the self-adjoint operator $\mathscr{F}_{\varepsilon} \in \mathscr{L}(Z)$ defined by

$$
\left(\mathscr{F}_{\varepsilon} a\right)_{n}=-\mathrm{e}^{-\lambda_{n}(T+\varepsilon)} a_{n} \quad(a \in Z, n \geqslant 0) .
$$

Applying Proposition 2.3 to the sequence $\left\{a_{n} \mathrm{e}^{-\varepsilon \lambda_{n}}\right\}_{n=0}^{\infty}$, we obtain that the bound

$$
\left\|\mathscr{F}_{\varepsilon}^{*} a\right\|_{Z} \ll \frac{\mathrm{e}^{c / T^{g}}}{\sqrt{T}}\left\|\mathscr{G}_{\varepsilon}^{*} a\right\|_{Y}
$$

holds for every $c>h^{g h} g^{-g^{2}} d_{1}^{h}$. Note that the implicit constant above does not depend on $\varepsilon$. By Lemma 2.1 with $W=Z$, it follows that there exists $\mathscr{H}_{\varepsilon} \in \mathscr{L}(Z, Y)$ such that $\mathscr{G}_{\varepsilon} \mathscr{H}_{\varepsilon}=\mathscr{F}_{\varepsilon}$. Moreover, $\left\|\mathscr{H}_{\varepsilon}\right\| \ll \mathrm{e}^{c / T^{g}} T^{-1 / 2}$ where the implied constant is independent of $\varepsilon>0$. Thus, for any $a \in Z$, we have

$$
\left(\mathscr{F}_{\varepsilon} a\right)_{n}=-a_{n} \mathrm{e}^{-\lambda_{n}(T+\varepsilon)}=\left(\mathscr{G}_{\varepsilon} \mathscr{H}_{\varepsilon} a(t)\right)_{n}=\int_{0}^{T}\left\langle\mathscr{H}_{\varepsilon} a(t), \mathrm{e}^{-\lambda_{n}(t+\varepsilon)} \psi_{n}\right\rangle_{U} \mathrm{~d} t \quad(n \geqslant 0) .
$$

Dividing through by $\mathrm{e}^{-\lambda_{n} \varepsilon}$, we see that $H_{T}:=\mathscr{H}_{\varepsilon}$ satisfies $(2.12)$ and (2.13).

Remark 2.5. Albeit our proof of Theorem 1.2 (see Sect. 3) only appeals to assumption (H2), we stated Proposition 2.4 in a more general form: indeed, its validity under assumption $(H 1)$ is of independent interest. This latter hypothesis can also be used for systems governed by diffusion equations (for instance in the proof of Cor. 1.6) since condition (i) in $(H 1)$ is satisfied when $-A_{0}$ is the Dirichlet Laplacian; this follows from Weyl's formula - in fact a simpler classical result (see, for instance [11], Prop. 3.6.9) suffices. 


\section{Proof of the main Results}

We first show that Theorem 1.2 is a simple consequence of Proposition 2.4.

Proof of Theorem 1.2. Set $a_{n}:=\left\langle z, \varphi_{n}\right\rangle(n \geqslant 0)$. From (1.3) and (1.6), we see that the null-controllability of (1.1) in time $T$ is equivalent to the existence, for every $z \in X$, of a function $u \in L^{2}([0, T], U)$ such that

$$
a_{n} \mathrm{e}^{-\lambda_{n} T}+\int_{0}^{T} \mathrm{e}^{-\lambda_{n}(T-t)}\left\langle u(t), B^{*} \varphi_{n}\right\rangle_{U} \mathrm{~d} t=0 \quad(n \geqslant 0) .
$$

Now Proposition 2.4 implies that the function

$$
u(t):=\left(H_{T} a\right)(T-t) \quad(t \in[0, T]),
$$

satisfies the above condition. Consequently, (1.1) is null-controllable in any time $T>0$. Finally, the cost estimate (1.8) readily follows from (2.13).

Our proof of Theorem 1.5 is based on the following result of Turán [12] - see also Lemma 1 in Montgomery [9], p. 89.

Lemma 3.1. Let $N \in \mathbb{N}^{*}, \Lambda:=[-N, N] \cap \mathbb{Z}$ and let $\left\{a_{n}\right\}_{n \in \Lambda}$ be a complex sequence. Put

$$
f(x):=\sum_{n \in \Lambda} a_{n} \mathrm{e}^{\mathrm{i} n x} \quad(x \in \mathbb{R})
$$

Then, for every subinterval $I$ of $[-\pi, \pi]$ with length $|I|=4 \pi \mathrm{e} L$, we have

$$
\sup _{x \in I}|f(x)| \geqslant L^{2 N} \max _{x \in[-\pi, \pi]}|f(x)| \text {. }
$$

This result opens the way to a comparison statement between the $L^{2}$-norms of a trigonometric polynomial over the full torus and over a restricted interval.

Proposition 3.2. With the notation in Lemma 3.1, we have

$$
\int_{I}|f(x)|^{2} \mathrm{~d} x \geqslant \frac{L^{6 N}}{8 \sqrt{2} N^{3 / 2}} \sum_{n \in \Lambda}\left|a_{n}\right|^{2} .
$$

Proof. Let $x_{0} \in I$ be such that $\left|f\left(x_{0}\right)\right|=\|f\|_{L^{\infty}(I)}$. By the Cauchy-Schwarz inequality, we have

$$
\left|f^{\prime}(x)\right| \leqslant N^{3 / 2} \sqrt{2 \sum_{n \in \Lambda}\left|a_{n}\right|^{2}} \leqslant \sqrt{2} N^{3 / 2}\|f\|_{L^{\infty}[-\pi, \pi]} \quad(x \in \mathbb{R}) .
$$

Set

so that it follows from (3.2) that

$$
h:=\frac{L^{2 N}}{\sqrt{8} N^{3 / 2}}
$$

$$
h\left|f^{\prime}(x)\right| \leqslant \frac{1}{2}\|f\|_{L^{\infty}[-\pi, \pi]} L^{2 N} \quad(x \in \mathbb{R}),
$$

and in turn, from Lemma 3.1,

$$
h \leqslant \frac{\left|f\left(x_{0}\right)\right|}{2\left\|f^{\prime}\right\|_{L^{\infty}[-\pi, \pi]}} .
$$


The above inequality implies that

$$
\min _{\left|x-x_{0}\right| \leqslant h}|f(x)| \geqslant \frac{1}{2}\left|f\left(x_{0}\right)\right| .
$$

Moreover, since $h<2 \pi \mathrm{e} L$, at least one of the intervals $\left[x_{0}-h, x_{0}\right]$ and $\left[x_{0}, x_{0}+h\right]$ is contained in $I$. Therefore

$$
\int_{I}|f(x)|^{2} \mathrm{~d} x \geqslant \frac{1}{4} h\left|f\left(x_{0}\right)\right|^{2} .
$$

Appealing to Lemma 3.1 again and using (3.3), it follows that

$$
\int_{I}|f(x)|^{2} \mathrm{~d} x \geqslant \frac{L^{6 N}}{4 \sqrt{8} N^{3 / 2}}\|f\|_{L^{\infty}[-\pi, \pi]}^{2} \geqslant \frac{L^{6 N}}{4 \sqrt{8} N^{3 / 2}} \sum_{n \in \Lambda}\left|a_{n}\right|^{2} .
$$

We now derive a straightforward corollary of the above proposition, adapted to sine or cosine series in several variables. More precisely, for every $m \geqslant 1$, we define

$$
\varphi_{\alpha, m}(x)=\left(\frac{2}{\pi}\right)^{m / 2} \prod_{1 \leqslant j \leqslant m} \sin \left(\alpha_{j} x_{j}\right) \quad\left(\alpha \in \mathbb{N}^{m}, x \in[0, \pi]^{m}\right),
$$

and

$$
\Phi \alpha, m(x)=\left(\frac{2}{\pi}\right)^{m / 2} \prod_{1 \leqslant j \leqslant m} \cos \left(\alpha_{j} x_{j}\right) \quad\left(\alpha \in \mathbb{N}^{m}, x \in[0, \pi]^{m}\right) .
$$

Corollary 3.3. Let $m, N \geqslant 1, \mathscr{D} \subset[0, N]^{m} \cap \mathbb{N}^{m}$, and $P(x):=\sum_{\alpha \in \mathscr{D}} b_{\alpha} \varrho_{\alpha, m}(x)\left(x \in \mathbb{R}^{m}\right)$ be a trigonometrical polynomial with complex coefficients, where either $\varrho_{\alpha, m}:=\varphi_{\alpha, m}$ for all $\alpha$ or $\varrho_{\alpha, m}:=\Phi_{\alpha, m}$ for all $\alpha$. Moreover, assume in the former case that $b_{\alpha}:=0$ whenever $\prod_{1 \leqslant j \leqslant m} \alpha_{j}=0$.

If $\mathscr{R}$ is a hyper-rectangle included in $[0, \pi]^{m}$ with measure $(4 \pi \mathrm{e})^{m} \vartheta>0$, we have

$$
\sum_{\alpha \in \mathscr{D}}\left|b_{\alpha}\right|^{2} \leqslant(4 \pi)^{m}\left(2 N^{3}\right)^{m / 2} \vartheta^{-6 N} \int_{\mathscr{R}}|P(x)|^{2} \mathrm{~d} x .
$$

Proof. We only consider the case $\left(\varrho_{\alpha, m}\right)=\left(\varphi_{\alpha, m}\right)$, since the other case is similar. We argue by induction upon $m$. When $m=1$, we have

$$
\mathrm{i} \sqrt{2 \pi} P(x)=\sum_{n \in \Lambda} a_{n} \mathrm{e}^{\mathrm{i} n x},
$$

with $\Lambda:=[-N, N] \cap \mathbb{Z}$ and $a_{n}=\operatorname{sgn}(n) b_{n}(n \in \Lambda)$. By Proposition 3.2, we thus obtain

$$
8 \pi \sqrt{2} N^{3 / 2} \vartheta^{-6 N} \int_{\mathscr{R}}|P(x)|^{2} \mathrm{~d} x \geqslant \sum_{n \in \Lambda}\left|a_{n}\right|^{2}=2 \sum_{1 \leqslant n \leqslant N}\left|b_{n}\right|^{2} .
$$

This is the required inequality for $m=1$.

Let $m \geqslant 2$ and assume $(3.7)$ holds for $m-1$. We may write $\mathscr{R}=\mathscr{S} \times I$ with $\mathscr{S} \subset[0, \pi]^{m-1},|\mathscr{S}|=4 \pi \mathrm{e} \tau$, $|I|=4 \pi \mathrm{e} \sigma$, and $\tau \sigma=\vartheta$. Moreover, setting

$$
\begin{aligned}
\mathscr{D}_{n} & :=\left\{\beta \in\left(\mathbb{N}^{*}\right)^{m-1}:(\beta, n) \in \mathscr{D}\right\} \quad(1 \leqslant n \leqslant N), \\
P_{n}(x) & :=\sum_{\beta \in \mathscr{D}_{n}} b_{\beta n} \varphi_{\beta, m-1}(x) \quad\left(1 \leqslant n \leqslant N, x \in \mathbb{R}^{m-1}\right),
\end{aligned}
$$


we have for all $x \in \mathbb{R}^{m-1}$, in view of (3.1),

$$
\int_{I}\left|P\left(x, x_{m}\right)\right|^{2} \mathrm{~d} x_{m}=\int_{I}\left|\sum_{1 \leqslant n \leqslant N} P_{n}(x) \varphi_{n, 1}\left(x_{m}\right)\right|^{2} \mathrm{~d} x_{m} \geqslant \frac{(\sigma / \mathrm{e})^{6 N}}{4 \pi \sqrt{2} N^{3 / 2}} \sum_{|n| \leqslant N}\left|P_{n}(x)\right|^{2},
$$

hence, by the induction hypothesis,

$$
\begin{aligned}
\int_{\mathscr{R}}|P(x)|^{2} \mathrm{~d} x & =\int_{\mathscr{S}} \int_{I}\left|P\left(x, x_{m}\right)\right|^{2} \mathrm{~d} x_{m} \mathrm{~d} x \geqslant \frac{\sigma^{6 N}}{4 \pi \sqrt{2} N^{3 / 2}} \sum_{|n| \leqslant N} \int_{\mathscr{S}}\left|P_{n}(x)\right|^{2} \mathrm{~d} x \\
& \geqslant \frac{(\tau \sigma)^{6 N}}{(4 \pi)^{m}\left(2 N^{3}\right)^{m / 2}} \sum_{|n| \leqslant N} \sum_{\beta \in \mathscr{D}_{n}}\left|b_{\beta n}\right|^{2} .
\end{aligned}
$$

Proof of Theorem 1.5. For the sake of simplicity, we select $\Omega=] 0, \pi^{[m}$. Let $-A_{0}$ be the Dirichlet Laplacian on $\Omega$. Then, the family $\left\{\varphi_{\alpha, m}: \alpha \in\left(\mathbb{N}^{*}\right)^{m}\right\}$ defined in (3.5) is an orthonormal basis of $L^{2}(\Omega)$ formed of eigenvectors of $-A_{0}^{\eta}$ on $\Omega$ and corresponding to the sequence of eigenvalues $\left(\lambda_{\alpha}\right)$ given by

$$
\lambda_{\alpha}:=\|\alpha\|^{2 \eta}=\left(\sum_{1 \leqslant k \leqslant m} \alpha_{k}^{2}\right)^{\eta} \quad\left(\alpha \in\left(\mathbb{N}^{*}\right)^{m}\right)
$$

Corollary 3.3 immediately furnishes constants $d_{0}, d_{1}$ such that, for every $\left\{a_{\alpha}\right\} \in \ell^{2}\left(\left(\mathbb{N}^{*}\right)^{n}, \mathbb{C}\right)$, we have

$$
\left(\sum_{\lambda_{\alpha}^{1 / 2 \eta} \leqslant \mu}\left|a_{\alpha}\right|^{2}\right)^{1 / 2} \leqslant d_{0} \mathrm{e}^{d_{1} \mu}\left\|\sum_{\lambda_{\alpha}^{1 / 2 \eta} \leqslant \mu} a_{\alpha} \varphi_{\alpha, m}\right\|_{L^{2}(\mathcal{O})} \quad(\mu>1) .
$$

Indeed, given any $\varepsilon>0$, we may select $d_{1}:=3(1+\varepsilon) m \log (4 \pi \mathrm{e})-3(1+\varepsilon) \log \left|\mathcal{O}^{*}\right|$, for any hyper-rectangle $\mathcal{O}^{*}$ included in $\mathcal{O}$ and then, since $d_{1} \geqslant 3 m \log (4 \mathrm{e})$,

$$
d_{0}:=2^{5 m / 4} \pi^{m / 2}\left(\frac{1}{4 \mathrm{e} \varepsilon \log (4 \mathrm{e})}\right)^{3 m / 4}=2^{-m / 4} \pi^{m / 2}\left(\frac{1}{\mathrm{e} \varepsilon \log (4 \mathrm{e})}\right)^{3 m / 4},
$$

in view of the elementary inequality $x^{3 m / 4} \leqslant(3 m / 4 \mathrm{e} \lambda)^{3 m / 4} \mathrm{e}^{\lambda x}$, which is valid for all $\lambda>0, m \geqslant 1, x \geqslant 0$. Since $B^{*} \varphi=\left.\varphi\right|_{\mathcal{O}}$ for every $\varphi \in L^{2}(\Omega)$, inequality (3.9) is precisely (1.7) with $\left.\gamma:=1 /(2 \eta) \in\right] 0,1[$.

We proceed similarly when $-A_{0}$ is the the Neumann Laplacian. The family $\left\{\Phi_{\alpha, m}: \alpha \in \mathbb{N}^{m}\right\}$ defined in (3.6) is an orthonormal basis of $L^{2}(\Omega)$ formed of eigenvectors of $-A_{0}^{\eta}$ on $\Omega$ and corresponding to the sequence of eigenvalues $\left(\lambda_{\alpha}\right)$ still given by (3.8), but with now $\alpha \in \mathbb{N}^{m}$. From this point on, the argument is unchanged.

Acknowledgements. The author are grateful to Luc Miller for drawing their attention on his submitted work [8] and for helpful discussions.

\section{REFERENCES}

[1] H.O. Fattorini and D.L. Russell, Exact controllability theorems for linear parabolic equations in one space dimension. Arch. Rational Mech. Anal. 43 (1971) 272-292.

[2] H.O. Fattorini and D.L. Russell, Uniform bounds on biorthogonal functions for real exponentials with an application to the control theory of parabolic equations. Quart. Appl. Math. 32 (1974) 45-69.

[3] A.V. Fursikov and O.Y. Imanuvilov, Controllability of Evolution Equations, Lect. Notes Ser. 34. Seoul National University Research Institute of Mathematics, Global Analysis Research Center, Seoul (1996). 
[4] G. Lebeau and L. Robbiano, Contrôle exact de l'équation de la chaleur. Comm. Partial Diff. Eq. 20 (1995) 335-356.

[5] G. Lebeau and E. Zuazua, Null-controllability of a system of linear thermoelasticity. Arch. Rational Mech. Anal. 141 (1998) $297-329$.

[6] S. Micu and E. Zuazua, On the controllability of a fractional order parabolic equation. SIAM J. Control Optim. 44 (2006) $1950-1972$

[7] L. Miller, On the controllability of anomalous diffusions generated by the fractional Laplacian. Math. Control Signals Systems 18 (2006) 260-271.

[8] L. Miller, A direct Lebeau-Robbiano strategy for the observability of heat-like semigroups. Preprint, available at http://hal. archives-ouvertes.fr/hal-00411846/en/ (2009).

[9] H.L. Montgomery, Ten lectures on the interface between analytic number theory and harmonic analysis, CBMS Regional Conference Series in Mathematics 84. Published for the Conference Board of the Mathematical Sciences, Washington (1994).

[10] T.I. Seidman, How violent are fast controls. III. J. Math. Anal. Appl. 339 (2008) 461-468.

[11] M. Tucsnak and G. Weiss, Observation and control for operator semigroups. Birkhäuser Advanced Texts: Basler Lehrbücher, Birkhäuser Verlag, Basel (2009).

[12] P. Turán, On a theorem of Littlewood. J. London Math. Soc. 21 (1946) 268-275. 